\title{
Global Initiative for Asthma report: How will new recommendations affect practice in Canada?
}

\author{
Marie-France Beauchesne PharmD, Catherine Lemiere MD MSc
}

Cite as: CMAJ 2020 April 27;192:E456-8. doi: 10.1503/cmaj.191445

$\mathbf{T}$ he 2019 Global Initiative for Asthma (GINA) report recommended the most radical change in asthma management among adults and adolescents aged 12 years and older in the last 30 years. ${ }^{1}$ The initiative, a global network of individuals, organizations and public health officials dedicated to the optimal treatment of asthma, performs a routine biannual literature review and has been updating its yearly reports since 2002. We discuss GINA's new recommendations for asthma management and its implications for clinicians' practice in Canada.

The recent GINA report recommended that short-acting $\beta_{2}$ agonists (e.g., salbutamol and terbutaline) no longer be used as the only treatment of mild intermittent asthma. The preferred choice of symptom reliever in any spectrum of asthma severity including mild intermittent - is the combination of a low-dose inhaled corticosteroid with the rapid-onset long-acting $\beta_{2}$ agonist formoterol. Formoterol is the only long-acting $\beta_{2}$ agonist indicated in asthma that shows a rapidity of onset comparable to that of salbutamol. ${ }^{2}$ As such, it was approved in combination with budesonide as maintenance and reliever therapy in moderate-tosevere asthma in 2007 and as an anti-inflammatory reliever therapy in 2019 in Canada.

The reason for the change recommended by GINA is that treatment with only short-acting $\beta_{2}$ agonists without a concomitant inhaled corticosteroid has been shown to be detrimental. For instance, the risk of death from asthma increases significantly when a patient uses 1.4 canisters (or more) of inhaled $\beta_{2}$ agonists per month. ${ }^{3}$ Furthermore, observational studies showed a higher rate of asthma-related deaths during the first 3 months after the discontinuation of an inhaled corticosteroid, with a relative risk of 4.6 (95\% confidence interval 1.1-19.1) compared with patients who continued treatment. ${ }^{3,4}$ In comparison to treatment with only short-acting $\beta_{2}$ agonists, regular treatment with an inhaled corticosteroid reduces the risk of asthma exacerbations by about $50 \%$, even in patients with mild asthma, ${ }^{5}$ and helps in preventing asthma-related deaths. ${ }^{4}$

The budesonide-formoterol maintenance and reliever strategy has long been shown to be superior to inhaled corticosteroid monotherapy or a combination of inhaled corticosteroid and long-acting $\beta_{2}$ agonist in association with as-needed short-

\section{KEY POINTS}

- As-needed low-dose inhaled corticosteroid combined with the rapid-onset long-acting $\beta_{2}$ agonist formoterol is recommended as the preferred maintenance and/or reliever therapy in adults with mild intermittent asthma.

- Given that treatment with only a short-acting $\beta_{2}$ agonist without a concomitant inhaled corticosteroid has been shown to be detrimental, the authors of the recent Global Initiative for Asthma (GINA) report wish to ensure that patients, particularly those who struggle to adhere to daily treatment, receive a minimum dose of an inhaled corticosteroid.

- For patients who manage to adhere well to regular inhaled corticosteroid treatment - with or without long-acting $\beta_{2}$ agonist therapy - with good asthma control, there is no need to change their current treatment to as-needed low-dose inhaled corticosteroid-formoterol only.

- Further studies are required to assess the long-term safety and efficacy of GINA's new recommendation regarding the use of as-needed low-dose inhaled corticosteroid-formoterol in practice.

acting $\beta_{2}$ agonists in reducing the risk of asthma exacerbations, with similar asthma control. ${ }^{6}$ A recent randomized controlled trial (RCT) showed that use of as-needed low-dose budesonideformoterol therapy reduced asthma exacerbations to a similar extent as regular treatment with low-dose inhaled corticosteroid (budesonide) and as-needed short-acting $\beta_{2}$ agonists in mild asthma. ${ }^{7}$ In this study, the group who received as-needed budesonide-formoterol used about a quarter of the median daily dose of the inhaled corticosteroid and had a similar annualized rate of severe exacerbations when compared with the group who received daily low-dose budesonide.

Although a regular low dose of inhaled corticosteroid reduces asthma mortality ${ }^{4}$ and seems superior to an as-needed low dose of the budesonide-formoterol therapy in controlling asthma symptoms, ${ }^{8}$ in practice, patients find it difficult to adhere to a daily regimen. For example, about $60 \%$ of pediatric and adult patients with asthma followed in 3 Quebec asthma clinics had suboptimal adherence to daily inhaled corticosteroid. ${ }^{9}$ Therefore, the GINA strategy proposes a pragmatic 
approach in recommending as-needed low-dose budesonideformoterol maintenance therapy in mild intermittent asthma. This treatment is also recommended as the preferred reliever medication for patients across the spectrum of asthma severity.

Furthermore, GINA emphasizes the importance of using the combination of a low-dose inhaled corticosteroid and formoterol from the onset of therapy to avoid overreliance on short-acting $\beta_{2}$ agonists only. Although this approach has its merits, it raises several challenges when applied in a clinical setting. The Novel START ${ }^{10}$ (Novel Symbicort Turbuhaler Asthma Reliever Therapy) controlled trial found no clinically significant differences in airway inflammation, based on fractional nitric oxid concentration in exhaled breath values, when comparing as-needed low-dose budesonide-formoterol with daily lowdose budesonide. However, no longitudinal study has compared airway remodelling several years after using an as-needed inhaled corticosteroid-formoterol treatment compared with a daily inhaled corticosteroid maintenance therapy. Nor has the effectiveness of the as-needed inhaled corticosteroidformoterol strategy compared with daily inhaled corticosteroid therapy been assessed in patients who cannot perceive their asthma symptoms well.

Daily low-dose budesonide achieved better asthma control and greater improvement in forced expiratory volume in $1 \mathrm{sec}-$ ond $\left(\mathrm{FEV}_{1}\right)$ than as-needed budesonide-formoterol treatment in an RCT. ${ }^{8}$ However, a real-world study, in which patients' adherence to treatment was lower than that in the trial, found no difference in asthma control between the 2 strategies. ${ }^{10}$

Canadian clinicians should note that the use of as-needed lowdose budesonide-formoterol in association with other combinations of inhaled corticosteroid and long-acting $\beta_{2}$ agonist, such as fluticasone propionate-salmeterol, fluticasone furoate-vilanterol or mometasone-formoterol, is not recommended, as the efficacy and safety of such a strategy has not been studied. Moreover, a strategy that used 2 combination treatments would likely be costlier than using budesonide-formoterol for both as-needed and maintenance treatment, as well as inconvenient since patients would need to learn how to use 2 different devices.

For many years, emphasis on the importance of taking daily inhaled corticosteroid therapy to reduce chronic airway inflammation, as opposed to taking the medication only when symptoms manifest, has dominated asthma education. Should the messaging now change? Since the regular use of an inhaled corticosteroid remains the most effective strategy in the improvement and maintenance of asthma control, the old message is still valid. The intent of the recent GINA recommendation is simply to ensure that patients receive a minimum dose of an inhaled corticosteroid, including those who find it difficult to adhere to recommended treatment; the new recommendation should not be misinterpreted as a disavowal of the importance of regular treatment with an inhaled corticosteroid. For patients who manage to adhere well to regular inhaled corticosteroid therapy with good symptom control, there is no need to change their current treatment to as-needed low-dose inhaled corticosteroid-formoterol only.

Practitioners may now wonder if they should step down the therapy of patients with well-controlled asthma from a combi- nation of daily inhaled corticosteroid and long-acting $\beta_{2}$ agonist to a combination of as-needed inhaled corticosteroid and formoterol, but this has not yet been studied. It must be emphasized that the recommendation to use as-needed low-dose inhaled corticosteroid-formoterol as a controller therapy applies only to patients who are at least 12 years of age and who have mild asthma; currently, when asthma is well controlled for at least 3 months, the step down from the regular use of a moderate-to-high dose of an inhaled corticosteroid-long-acting $\beta_{2}$ agonist consists of a reduction of the inhaled corticosteroid dose by $50 \%$ with a daily inhaled corticosteroid-long-acting $\beta_{2}$ agonist treatment. ${ }^{1}$

Clinicians may also have questions regarding stepping up therapy. When asthma control is not optimal with an as-needed inhaled corticosteroid-formoterol treatment, should clinicians initiate daily inhaled corticosteroid or inhaled corticosteroidformoterol treatment? The optimal step-up strategy for improving asthma control from as-needed low-dose inhaled corticosteroid-formoterol to another therapy has not yet been studied. No data currently support one strategy over another as the most cost-effective step-up therapy in patients whose symptoms are not controlled with as-needed low-dose budesonide-formoterol.

Budesonide-formoterol is the only inhaler that has received approval from Health Canada as an anti-inflammatory reliever therapy in patients at least 12 years of age with mild, moderate and severe asthma. Although the combination mometasoneformoterol is available in Canada and sometimes prescribed as a maintenance and reliever therapy, it is not indicated and has not been studied as a reliever therapy. The budesonide-formoterol combination inhaler is not currently covered by some public drug insurance plans when a daily inhaled corticosteroid has not been previously prescribed. However, in our estimation, based on the mean use of a 0.5 inhalation per day of as-needed lowdose budesonide-formoterol described in previous trials, ${ }^{6,7}$ it is likely that the long-term cost of the medication would be less when compared with that of a daily inhaled corticosteroid with short-acting $\beta_{2}$ agonists as needed.

The 2019 GINA report proposes a new pragmatic approach to the treatment of mild asthma in patients 12 years and older, particularly for those who struggle to adhere to daily inhaled corticosteroid treatment. However, patients who manage well on current treatment should be encouraged to continue with daily maintenance therapy. Asthma education remains an important strategy to optimize patients' ability to adhere to treatment and control symptoms. Further studies are required to assess the long-term safety and efficacy of GINA's new recommendation in practice.

\section{References}

1. Global strategy for asthma management and prevention: updated 2019. Fontana (WI): Global Initiative for Asthma; 2019. Available: https://ginasthma.org/ wp-content/uploads/2019/06/GINA-2019-main-report-June-2019-wms.pdf (accessed 2020 March).

2. Seberová E, Andersson A. Oxis (formoterol given by Turbuhaler) showed as rapid an onset of action as salbutamol given by a pMDI. Respir Med 2000;94: 607-11. 
3. Suissa S, Ernst P, Boivin J, et al. A cohort analysis of excess mortality in asthma and the use of inhaled B-agonists. Am J Respir Crit Care Med 1994;149: 604-10.

4. Suissa S, Ernst P, Benayoun S, et al. Low-dose inhaled corticosteroids and the prevention of death from asthma. N Engl J Med 2000;343:332-6.

5. Busse WW, Pedersen S, Pauwels RA, et al. The Inhaled Steroid Treatment As Regular Therapy in Early Asthma (START) study 5-year follow-up: effectiveness of early intervention with budesonide in mild persistent asthma. J Allergy Clin Immunol 2008;121:1167-74.

6. O'Byrne PM, Bisgaard H, Godard PP, et al. Budesonide/formoterol combination therapy as both maintenance and reliever medication in asthma. $\mathrm{Am} \mathrm{J}$ Respir Crit Care Med 2005;171:129-36.

7. Bateman ED, Reddel HK, FitzGerald JM. As-needed budesonide-formoterol in mild asthma. N Engl J Med 2018;379:898.

8. Bateman ED, Reddel HK, O'Byrne PM, et al. As-needed budesonide-formoterol versus maintenance budesonide in mild asthma. N Engl J Med 2018;378: 1877-87.

9. Blais L, Kettani FZ, Forget A, et al. Assessing adherence to inhaled corticosteroids in asthma patients using an integrated measure based on primary and secondary adherence. Eur J Clin Pharmacol 2017;73:91-7.

10. Beasley R, Holliday M, Reddel HK, et al. Controlled trial of budesonideformoterol as needed for mild asthma. N Engl J Med 2019;380:2020-30.
Competing interests: Marie-France Beauchesne has received grant support and speaker fees from Novartis in the last 3 years. Catherine Lemiere has received grant support from AstraZeneca and Teva Innovation, consultancy fees from AstraZeneca, GlaxoSmithKline, Metapharm, Novartis, Sanofi and Teva Innovation in the last 3 years. Catherine Lemiere currently sits on the executive board of the Canadian Thoracic Society.

This article has been peer reviewed.

Affiliations: Faculté de pharmacie (Beauchesne) and Faculté de médecine (Lemiere), Université de Montréal, Montréal, Que.; Research Center and Pharmacy Department (Beauchesne), Centre intégré universitaire de santé et de services sociaux (CIUSSS) de l'Estrie - Centre hospitalier universitaire de Sherbrooke, Sherbrooke, Que.; Hôpital du Sacré-Coeur de Montréal (Lemiere), CIUSSS du Nord-de-l'île-de-Montréal, Montréal, Que.

Contributors: Marie-France Beauchesne and Catherine Lemiere participated equally in the writing of the article and its revision. Both approved the final version to be published and agree to be accountable for all aspects of this work.

Acknowledgements: The authors thank Abou-Atmé and James Hatch for reviewing the manuscript.

Correspondence to: Catherine Lemiere, catherine.lemiere@ umontreal.ca 